Published in final edited form as:

Mol Nutr Food Res. 2016 July ; 60(7): 1544-1554. doi:10.1002/mnfr.201501068.

\title{
Polyphenol Rich Olive Oils improve Lipoprotein Particle Atherogenic Ratios and Subclasses Profile: A Randomized, Crossover, Controlled Trial
}

\author{
Sara Fernández-Castillejo, MS, PhD S 1 , Rosa-Maria Valls, $\mathrm{PhD}^{1}$, Olga Castañer, $\mathrm{PhD}^{2}$, \\ Laura Rubió, $\mathbf{P h D}^{1,3}$, Úrsula Catalán, $\mathbf{P h D}^{1}$, Anna Pedret, $\mathbf{P h D}^{1}$, Alba Macià, $\mathbf{P h D}^{3}$, Maureen \\ L Sampson, BS ${ }^{4}$, María-Isabel Covas, DPharm, PhD $^{2}$, Montserrat Fitó, PhD², Maria-José \\ Motilva, $\mathbf{P h D}^{3}$, Alan T Remaley, MD, $\mathrm{PhD}^{4,5}{ }^{,}$, and Rosa Solà, $\mathbf{M D}, \mathrm{PhD}^{1}{ }^{, *}$ \\ ${ }^{1}$ Research Unit on Lipids and Atherosclerosis; Hospital Universitari Sant Joan; Institut \\ Investigació Sanitària Pere Virgili; NFOC group; Facultat de Medicina i Ciències de la Salut; \\ Universitat Rovira i Virgili; CIBERDEM; Reus, Spain \\ ${ }^{2}$ Cardiovascular Risk and Nutrition Research group; IMIM- Institut Hospital del Mar \\ d'Investigacions Mèdiques; CIBEROBN; Barcelona, Spain \\ ${ }^{3}$ Food Technology Department; Agrotecnio Center; University of Lleida; Lleida, Spain \\ ${ }^{4}$ Department of Laboratory Medicine, Clinical Center, National Institutes of Health, Bethesda, MD, \\ USA \\ ${ }^{5}$ Lipoprotein Metabolism Section, Cardio-Pulmonary Branch, National Heart, Lung and Blood \\ Institute, National Institutes of Health; Bethesda, MD, USA
}

\begin{abstract}
Scope-Lipoprotein particle measures performed by nuclear magnetic resonance (NMR), and associated ratios, may be better markers for atherosclerosis risk than conventional lipid measures. The effect of two functional olive oils, one enriched with its polyphenols (FVOO, $500 \mathrm{ppm}$ ), and the other (FVOOT) with them (250 ppm) and those of thyme (250 ppm), versus an standard virgin olive oil (VOO), on lipoprotein particle atherogenic ratios and subclasses profiles was assessed.
\end{abstract}

Methods and Results-In a randomized, double-blind, crossover, controlled trial, 33 hypercholesterolemic individuals received $25 \mathrm{~mL} /$ day of VOO, FVOO, and FVOOT. Intervention periods were of 3-weeks separated by 2-week washout periods. Lipoprotein particle counts and subclasses were measured by NMR. Polyphenols from olive oil and thyme modified the

Corresponding authors: Rosa Solà, MD, PhD, Facultat de Medicina, Universitat Rovira Virgili, C/ Sant Llorenç, 21, 43201 Reus, Spain, Tel: (+34) 977759369 / (+34) 609906 991, FAX: (+34) 9777593 22, rosa.sola@urv.cat, Alan T. Remaley, MD, PhD, National Institutes of Health, Building 10-2C433, 10 Center Drive, SC1666, Bethesda, MD 20892, USA., Tel: (301) 402-9796, FAX: (301) 402-1885, aremaley1@nhlbi.nih.gov.

Co-Corresponding authors.

Conflict of interest: there are no competing interests to declare, as there is no relationship with industry that should be disclosed. Author contributions

SFC, MIC, ATR and RS have designed, analysed and interpreted the data, as well as draft the manuscript and revised it critically. MF and MJM have designed, analysed and contributed in the data interpretation. RMV, OC, LR, UC, AP, AM, MLS have contributed in the analysis of the data and also revised the manuscript critically. 
lipoprotein subclasses profile and decreased the total LDL particle/total HDL particle (HDL-P), small HDL/large HDL, and HDL-cholesterol/HDL-P ratios, and decreased the lipoprotein insulin resistance index (LP-IR) $(\mathrm{P}<0.05)$.

Conclusions-Olive oil polyphenols, and those from thyme provided benefits on lipoprotein particle atherogenic ratios and subclasses profile distribution. Polyphenol-enriched olive oil is a way of increasing the olive oil healthy properties while consuming the same amount of fat, as well as a useful and complementary tool for the management of cardiovascular risk individuals.

\section{Keywords}

HDL-C/HDL-P and LDL-P/HDL-P ratios; lipoprotein subclasses; olive oil; polyphenols; thyme

\section{Introduction}

In experimental and human studies, polyphenol-rich foods have shown to improve the lipid cardiovascular risk profile. Thyme extracts decrease total and LDL cholesterol (LDL-C) and increase HDL-C cholesterol (HDL) in animal models [1], as well as reduce the susceptibility to in vitro LDL oxidation [2]. Plant extracts, such as those from red yeast rice, sugar canederived policosanols, and artichoke leaf, have also been shown to have a LDL-C lowering effect in subjects with moderate hyperlipidemia [3]. Cocoa flavonols increase the HDL-C in human studies [4,5]. Data from the NHANES study showed that urinary enterolignan concentrations were positively associated with serum HDL-C and negatively associated with serum triglycerides (TG) in U.S. adults [6]. In the EUROLIVE (The effect of olive oil on oxidative damage on European populations) study, sustained consumption of polyphenolrich olive oils have been shown to: 1) increase HDL-C; 2) reduce total cholesterol (TC)/ HDL-C and LDL-C/HDL-C ratios; 3) decrease in vivo lipid oxidative markers, such as oxidized LDL [7,8]; and 4) increase HDL cholesterol efflux from macrophages [9]. Supporting these data, a functional olive oil enriched with its polyphenols has also been shown to increase the expression of cholesterol-efflux-related genes [10].

A relatively new approach for the measurement of lipoproteins is to use nuclear magnetic resonance (NMR), which provide not only total particle counts of the major lipoprotein fractions but also their mean size and size subclass distribution [11]. In several studies the measurement of LDL particles (LDL-P) by NMR was more effective than those of the cholesterol content of the LDL, or apolipoprotein (Apo) B100 concentrations, as a positive risk marker for CHD [12]. Results from large studies such as MESA (Multi-Ethnic Study of Atherosclerosis) [13], JUPITER [14], and HPS (Heart Protection Study) [15], also showed that measurement of HDL particles (HDL-P) appeared to be better than HDL-C as cardiovascular disease risk marker. Also within the MESA study, the LDL-P/HDL-P ratio was shown to be an independent risk factor for CHD [16].Recently, it has been described that cholesterol-overloaded particles, reflected in the HDL-C/HDL-P ratio, are independently associated with the progression of carotid atherosclerosis in a cardiovasculardisease-free population [17].

The aim of this work was to assess the effect of two functional olive oils, one enriched with its polyphenols, and the other with them and those of thyme, on the NMR lipoprotein 
particle profile and atherogenic ratios. We hypothesized that besides its positive effect on the standard lipid profile, the consumption of a polyphenol-rich olive oil rich diet may also improve cardiovascular risk parameters, as determined by NMR, and that this may provide an alternative way to monitor such treatment.

\section{Materials and Methods}

\subsection{Olive oil characteristics}

A natural virgin olive oil (VOO, $80 \mathrm{ppm}$ of phenolic compounds (PC)) was used as a control condition, and as a matrix to prepare a functional VOO (FVOO; $\mathrm{PC}=500 \mathrm{ppm})$ by enrichment of the $\mathrm{VOO}$ with its own PC. A second functional olive oil was prepared by enrichment of the VOO both with its PC and those of thyme (FVOOT; PC=500ppm (250 $\mathrm{ppm}$ from VOO and $250 \mathrm{ppm}$ from thyme)). Olive oils did not differ in fat and micronutrient composition, with the exception of the phenolic content (Supporting Information Table S1).

\subsection{Study subjects}

Hypercholesterolemic ( $\mathrm{TC}>200 \mathrm{mg} / \mathrm{dL}$ ) individuals were recruited from newspaper and university advertisements. Volunteers were preselected when their clinical record, physical examination, and blood pressure were within a predefined normal range and the candidate was non-smoker. Next, complete blood count, routine biochemical laboratory analyses, and urinary dipstick tests were performed. We included candidates with values, other than total and LDL-C, within the reference range for routine haematological and biochemical analyses. Exclusion criteria were the following: LDL-C $\geq 190 \mathrm{mg} / \mathrm{dL}, \mathrm{TG} \geq 350 \mathrm{mg} / \mathrm{dL}$, fasting blood glucose $>126 \mathrm{mg} / \mathrm{dL}$, plasma creatinine levels $>1.4 \mathrm{mg} / \mathrm{dL}$ for women and $>1.5 \mathrm{mg} / \mathrm{dL}$ for men, body mass index (BMI) $>35$, smokers ( $>1$ cigarrete/day), athletes with physical activity (>3000 METS.min/day), hypertension, multiple allergies, intestinal diseases, chronic diseases (i.e diabetes, cardiovascular, etc.), or other conditions that would impair the adherence to the study. All participants provided written informed consent, and the institutional ethic committee (CEIC-IMAS 2009/3347/I) approved the protocol.

\subsection{Design and study procedure}

This work was conducted in the frame of the VOHF (Virgin Olive Oil and HDL Functionality) Study. The trial was a randomized, crossover, double-blind, controlled study. We randomly assigned participants consecutively to 1 of 3 sequences of raw olive oil administration. Participants received a daily dose of $25 \mathrm{~mL}(22 \mathrm{~g}$ ) of raw VOO, FVOO, or FVOOT. Administration sequences were: FVOO,FVOOT,VOO (sequence 1, n=11); FVOOT,VOO,FVOO (sequence 2, n=11); and VOO,FVOO,FVOOT (sequence 3, n=11). Random allocation to each sequence was performed using a specific software developed at the Institut Hospital del Mar d'Investigacions Mèdiques (IMIM). Three-week interventions were preceded by 2-week washout periods, in which participants consumed a common olive oil, a mixture of VOO and refined olive oil, with a very low phenolic content, and avoided other types of raw fats as well as olive tables consumption. We chose the 2-week washout period to reach equilibrium in the plasma lipid profile, because longer intervention periods with fat-rich diets did not modify the lipid concentrations [18]. Daily doses of $25 \mathrm{~mL}$ of olive oil were blindly prepared in containers and delivered to the participants at the 
beginning of each intervention period. Containers were assigned a code number concealed from participants and investigators, and disclosed only after statistical analyses completion. We instructed participants to return the 21 containers at the end of each intervention period so that the daily amount of unconsumed olive oil could be registered. 24h-urine and blood samples were collected at a fasting state at the start of the study and before and after each treatment. Plasma EDTA and serum samples were obtained by whole blood centrifugation and preserved at $-80^{\circ} \mathrm{C}$. The present clinical trial was conducted in accordance with the Helsinki Declaration and the Good Clinical Practice for Trials on Medical Products in the European Community (http://ec.europa.eu/health/files/eudralex/vol-10/3cc1aen_en.pdf). The protocol is registered with the International Standard Randomized Controlled Trial register (www.controlled-trials.com:ISRCTN77500181).

\subsection{Dietary adherence}

We measured 24h-urinary hydroxytyrosol-sulfate and thymol-sulfate, before and after each intervention period as biomarkers of adherence to the FVOO and FVOOT interventions, respectively. Measurements were performed by HPLC-ESI-MS/MS [19]. A 3-day dietary record was administered by the participants at baseline and before and after each intervention-period. Participants were asked to avoid a high intake of foods rich in antioxidants (i.e. vegetables, legumes, fruits, etc.). A nutritionist personally advised participants to replace all types of habitually consumed raw fats with the olive oils catered, and to limit their rich-polyphenol food consumption.

\subsection{Outcomes and data collection}

Main outcome measures were changes in lipoprotein particle atherogenic ratios and subclasses. We assessed outcome measures at the beginning of the study (baseline) and before (pre-intervention) and after (post-intervention) each olive oil intervention period. Anthropometric variables were also recorded. Blood pressure was measured with a mercury sphygmomanometer after at least a 10-minute rest in the seated position. Physical activity was recorded at baseline and at the end of the study and assessed by the MLTPAQ, which has been validated for its use in Spanish men and women [20, 21]. Plasma glucose, TC, and TG were measured using standard enzymatic automated methods and ApoAI and ApoB100 by immunoturbidimetry, in a PENTRA-400 autoanalyzer (ABX-Horiba Diagnostics, Montpellier, France). HDL-C was measured by an accelerator selective detergent method (ABX-Horiba Diagnostics, Montpellier, France). LDL-C was calculated by the Friedewald equation.

Serum samples were shipped to the National Heart, Lung and Blood Institute, National Institutes of Health (NIH; Bethesda, MD, USA). Lipoprotein subclasses measurement was performed by NMR in a Vantera clinical spectrometer, produced by LipoScience (Raleigh, NC, U.S.A.). The NMR LipoProfile test by LipoScience involves measurement of the 400MHz proton NMR spectrum of samples and uses the characteristic signal amplitude of the lipid methyl group broadcast by every lipoprotein subfraction as the basis for quantification. NMR using the LipoProfile-3 algorithm was performed to quantify the average particle size and concentrations of VLDL, LDL, and HDL. Subparticle concentrations were determined for 3 VLDL subclasses (large or chylomicrons: >60nm; 
medium: 35-60nm; and small: 27-35nm); 3 LDL subclasses (intermediate-density lipoprotein (IDL): $23-27 \mathrm{~nm}$; large: $21.2-23 \mathrm{~nm}$; and small: $18-21.2 \mathrm{~nm}$ ); and $3 \mathrm{HDL}$ subclasses (large: $8.8-13 \mathrm{~nm}$; medium: $8.2-8.8 \mathrm{~nm}$; and small: 7.3-8.2nm) [22]. LP-IR, a lipoprotein particle-derived measure of insulin resistance [23] was also assessed.

\subsection{Sample size and power analysis}

Assuming a dropout rate of $15 \%$ and a Type I error of 0.05 (2-sided), a sample size of 32 individuals allows at least $80 \%$ power to detect a statistically significant difference among groups of $1.5 \mu \mathrm{mol} / \mathrm{L}$ in the total HDL particle number. The population standard deviation of this variable is $4.4 \mu \mathrm{mol} / \mathrm{L}$ [17].

\subsection{Statistical Analyses}

The normality of variables was assessed by the Kolmogorov-Smirnov test. We used the Kruskal-Wallis test or 1-factor analysis of variance (ANOVA), as appropriate, with Bonferroni correction for multiple comparisons, to determine differences in baseline characteristics. Carryover effect was discarded by testing a period-by-treatment interaction term in general linear models. Comparisons among changes were carried out by a covariance model. Age, gender, sequence of olive oil administration, LDL-C, and baseline values were the covariates. The P value for a trend among oils: from VOO to FVOO to FVOOT was assessed. Statistical significance was defined as a $P$ value less than 0.050 for a 2-sided test. We performed analyses by using SPSS for Windows, version 22 (IBM corp., Armonk, NY, USA).

\section{Results}

\subsection{Characteristics of the study participants}

From 62 subjects who were assessed for eligibility, 29 were excluded. Finally, 33 eligible participants (19 men, 14 women) entered the study. One participant in each sequence discontinued the intervention (Fig.1). We could not identify any adverse effects related to the olive oils intake. No significant differences in participants' baseline characteristics were observed among sequences of olive oil administration, with exception of the small HDL particles (s-HDL) which were slightly higher in sequence 3 versus 1 (Table 1). No changes in daily energy expenditure in leisure-time physical activity were observed from the beginning to the end of the study (data not shown).

\subsection{Dietary intake and adherence}

Table 2 shows the daily dietary intake after intervention periods. Diet was similar in all intervention groups. Participant adherence was good, as reflected in the changes in urinary hydroxytyrosol sulfate and thymol sulfate excretion after olive oil interventions (Fig.2). Hydroxytyrosol sulfate increased after FVOO $(\mathrm{P}<0.05)$. Thymol sulfate increased after FVOOT, the change reaching significance versus those of the other two olive oils $(\mathrm{P}<0.05)$. 


\subsection{Classical cardiovascular risk factors}

Functional olive oils consumption did not change either glucose levels or the classical cardiovascular lipid profile (TC, TG, LDL-C and HDL-C), nor ApoAI or ApoB100 concentrations versus VOO. An exception was a decrease in LDL-C after FVOO, which was significant versus changes after the other two olive oil interventions $(\mathrm{P}<0.05)$ (Table 3$)$. No changes were observed in blood pressure or BMI associated with the interventions.

\subsection{NMR lipoprotein particle counts and subclasses}

Total LDL-P, IDL-P and total ApoB100 containing lipoproteins concentrations decreased after FVOO, the decrease reaching significance versus changes after VOO and FVOOT $(\mathrm{P}<0.001)$. The decrease observed in small LDL particles after FVOO intervention was statistically significant $(\mathrm{P}<0.05)$ when compared with the changes observed after FVOOT intervention. LDL particle size decreased after FVOO intervention, the decrease reaching significance versus changes after the other interventions $(\mathrm{P}<0.05)$ (Table 3$)$.

Comparison among changes after interventions in HDL-related measures (Table 3) showed that both FVOO and FVOOT promoted an increase in large HDL (I-HDL) particles versus VOO $(\mathrm{P}<0.05)$. s-HDL particles decreased after FVOO intervention versus changes after VOO and FVOOT $(\mathrm{P}<0.05)$. Both functional olive oils increased HDL particle size when comparing with changes after the VOO intervention, the increase being higher after FVOO $(\mathrm{P}<0.05)$. Both functional olive oils decreased medium VLDL particles versus VOO intervention, the decrease being higher after FVOO $(\mathrm{P}<0.05)$. The average VLDL particle size decreased after FVOO intervention, reaching significance versus changes observed after the other two olive oil interventions $(\mathrm{P}<0.05)$.

\subsection{Atherogenic lipoprotein particle ratios and LP-IR}

Figure 3 shows the results obtained in the atherogenic lipoprotein particle ratios and LP-IR after FVOO and FVOOT versus control (VOO). The LDL-P/HDL-P ratio decreased after FVOO $(\mathrm{P}<0.05)$ versus VOO and FVOOT $(\mathrm{P}<0.05)$. Both functional olive oils also decreased the HDL-C/HDL-P and the s-HDL/1-HDL ratios $(\mathrm{P}<0.05)$. The decrease in sHDL/l-HDL after FVOO was higher than that after FVOOT $(\mathrm{P}<0.05)$. The LP-IR ratio also decreased after both functional olive oils $(\mathrm{P}<0.05)$.

\section{Discussion}

In this study, we assessed the effects of polyphenol-rich olive oils on lipoprotein particle atherogenic ratios and subclasses distribution, as determined by NMR. From our results, polyphenols from olive oil decreased LDL-C and the LDL-P/HDL-P ratio, and improved the lipoprotein subclasses by decreasing total ApoB100 containing lipoproteins, LDL and IDL particle concentration, as well as LDL and VLDL particle size. Both, polyphenols from olive oil, and combined equally with those of thyme, decreased medium VLDL particles, increased large HDL particles, decreased s-HDL/l-HDL and HDL-C/HDL-P ratios, and the lipoprotein insulin resistance index (LP-IR) $(\mathrm{P}<0.05)$. 
LDL-C, LDL-P, LDL size, and the LDL-P/HDL-P ratio have all been shown to be directly associated with the risk of coronary heart disease (CHD) [24, 25]. In the present work, olive oil polyphenols decreased all these cardiovascular risk biomarkers. The decrease in LDL-C observed is in agreement with that recently reported at postprandial state after extra-VOO consumption [26]. Polyphenols from green tea and from herbs have shown to have hypocholesterolemic effects lowering LDL-C in human [27] and animal models [28]. Mechanisms for explaining this effect are still unknown. The decrease in LDL-P after polyphenol-rich olive oil consumption observed in this study is also in agreement with our recent results from the EUROLIVE study with rich-polyphenol olive oil [29]. Concerning the decrease in LDL size, depletion of cholesteryl esters and triglyceride enrichment of LDL particles are associated with a decreased size, as well as an increased density of these particles, and these properties reduce their affinity for the LDL receptor [30]. As a consequence, small and dense LDL are likely to have an increased residence time in the circulation, making them more susceptible to oxidation or glycosylation, which considerably increases their atherogenic potential [31]. Olive oil polyphenols have shown to protect human LDL in vivo from oxidation [7]. In this sense, on November 2011, the European Food Safety Authority (EFSA) released a claim concerning the benefits of polyphenol-rich olive oil consumption protecting the LDL from oxidation [32]. Of all LDL particle biomarkers tested, the ratio LDL-P/HDL-P seems to have the strongest independent association with CHD, with significant net reclassification improvements in the AHA/ACC CHD risk scores [16].

To date the strong epidemiological association between HDL-C and CHD has failed to be translated into clinical benefit in terms of drug development. HDL, however, encompasses a heterogeneous population of lipoproteins that differ in shape, density, size, surface charge, and antigenicity that could exhibit differences in functionality irrespective of its cholesterol content. Studies reporting associations between HDL particle subclasses and CHD have been conflicting, and it is still unclear whether specific subclasses of HDL are more cardioprotective [33]. In most, but not all population studies [34], small HDL particles are considered to be more strongly associated with increased CHD risk than large HDL [35, 36]. High levels of small HDL particles and/or low levels of the large HDL ones are often present in CHD, ischemic stroke, and type-II diabetes mellitus [37-39]. In our study we observed a decrease in the s-HDL/l-HDL ratio after both functional olive oils consumption. Our results agree with those previously obtained in the VOHF study, when, by using gradient electrophoresis, an increase in the large less dense $\mathrm{HDL}\left(\mathrm{HDL}_{2}\right)$ and a decrease in the small denser $\mathrm{HDL}\left(\mathrm{HDL}_{3}\right)$ after both functional olive oils was observed, although significance was only reached for FVOOT [40]. Differences among results could be explained by the fact that different methods (NMR versus gradient electrophoresis) and scores for HDL subclasses classification were used.

A potentially important advance in cardiovascular biomarker testing related to HDL is the HDL-C/HDL-P ratio and is considered to be a potential new measure of HDL cardioprotective function $[17,41]$. This ratio indicates the enrichment of the HDL particle in cholesterol, and has been shown to be directly related with the atherosclerosis progression in cardiovascular-free individuals [17]. In our study, both functional olive oils decreased the HDL-C/HDL-P ratio versus a natural VOO. Thus, olive oil rich in its polyphenols and those 
from thyme were able to decrease the cholesterol enrichment of HDL. The most likely mechanism proposed to explain the benefits of this decrease is that cholesterol enrichment of HDL impairs its ability to promote the efflux of cholesterol from peripheral cells, considered to be one of the main HDL anti-atherogenic functions and inversely related to CVD risk. This cholesterol efflux impairment can be explained by the fact that these cholesteroloverloaded HDLs may act as donor more than as acceptors of cholesterol from peripheral tissues [42]. Moreover, HDL particles enriched in cholesterol may be less prone to be recognized by hepatic SR-BI receptors and thus, cholesterol clearance may be compromised. This fact can be explained by the assertion that myeloperoxidase enzyme oxidizes phospholipids and ApoA1 present in large, dense HDL [43]. Moreover, as reviewed by Lee et al., SR-BI have a lower binding affinity for oxidized phospholipids, blocking thus cholesteryl esters transfer from oxidized HDLs to hepatic cells [44]. In addition, cholesterolenriched HDLs become more pro-inflammatory, as well as more prone to be oxidised, which in turn impairs cholesterol efflux $[17,42] .$. In agreement with the results obtained in the present study, we also previously reported that olive oil polyphenols increase the efflux of cholesterol in vivo in humans [9], at least in part due via a transcriptomic effect [10]. Consumption of olive oils rich in polyphenols promoted a greater HDL stability, reflected in a triglyceride-poor core, and decreased the HDL oxidative status, through an increase in the olive oil polyphenol metabolites content in the lipoprotein [9].

Dyslipidemia among individuals with type 2 diabetes is characterized not only by high TG concentrations and low HDL-C concentrations, but also by decreases in the size of LDL and HDL particles and increases in the size of VLDL particles [45].In individuals with insulin resistance (IR) and diabetes, VLDL particle size is inversely associated with the glucose disposal rate (GRD) [46] the gold standard for assessing insulin sensitivity, and directly associated with incident hypertension [46]. In our study, FVOO consumption reduced the VLDL particle size in dyslypemic individuals versus the other olive oils, and both functional olive oils were effective, versus VOO, on reducing medium VLDL subclass concentration.

Larger VLDL particles have been associated with diabetes [45], and both large and medium VLDL concentrations with hypertension [46]. The American College of Endocrinology Task Force on the IR Syndrome states the clinical preventive value of identifying individuals with IR [47]. One measure to assess IR is the LP-IR index derived from lipoprotein NMR measurements. LP-IR is directly related with the HOMA index and inversely related with GRD, and has been proposed as a simple method for assessing the risk to develop a prediabetic or diabetic state [23].In agreement with all the other benefits derived from the consumption of the functional olive oils on the lipoprotein subclasses, both FVOO and FVOOT also decreased the LP-IR index in our dyslypemic individuals.

In summary, sustained consumption of VOO enriched with its PC (FVOO), or equally enriched with them and those of thyme (FVOOT), promoted benefits, particularly FVOO, on lipoprotein subclasses distribution versus a natural virgin olive oil (VOO). Compared with VOO consumption, both functional olive oils decreased the LP-IR index and the atherogenic ratios: HDL-C/HDL-P ands-HDL/l-HDL. FVOO also improved the LDL-P/HDL-P ratio. To the best of our knowledge this is the first time that a decrease in these atherogenic ratios associated to a dietary intervention has been reported. Our results provide first level evidence of the benefits of olive oil polyphenols and those from thyme on lipoprotein 
subclasses distribution and their associated ratios. Olive oil, a recognized healthy food, can not, however, be readily consumed in large quantities. Thus, polyphenol-enriched olive oil is a way of increasing its healthy properties while the same amount of fat is consumed. Data from this study provide further evidence to recommend polyphenol-rich olive oil consumption as a possibly useful and complementary tool for the management of cardiovascular risk individuals.

\section{Supplementary Material}

Refer to Web version on PubMed Central for supplementary material.

\section{Acknowledgments}

This study was supported by the Spanish Ministry of Education and Science, The VOHF Project (AGL2009-13517). Work done by ATR was supported by the intramural National Heart, Lung and Blood Institute of the National Institutes of Health, in Bethesda, MD. We thank NUPROAS HB for their substantial contribution interpreting the data and revising the manuscript critically.

\section{Abbreviations and Acronyms}

CHD

HDL-C

HPLC-ESI-MS/MS

JUPITER

LDL-C

LP-IR

MLTPAQ

NHANES coronary heart disease

high density lipoprotein cholesterol

high performance liquid chromatography-electrospray tandem mass spectrometry

Justification for the Use of Statins in Prevention: an Intervention Trial Evaluating Rosuvastatin

low-density lipoprotein cholesterol

Lipoprotein Insulin Resistance Index

Minnesota Leisure Time Physical Activity Questionnaire

National Health and Nutrition Examination Survey

\section{References}

1. Alamgeer, Akhtar M-S, Jabeen Q, Khan H-U, Maheen S, et al. Pharmacological evaluation of antihypertensive effect of aerial parts of Thymus linearis benth. Acta Pol. Pharm. 2014; 71:677682. [PubMed: 25272894]

2. Kulisić T, Krisko A, Dragović-Uzelac V, Milos M, Pifat G. The effects of essential oils and aqueous tea infusions of oregano (Origanum vulgare L. spp. hirtum), thyme (Thymus vulgaris L.) and wild thyme (Thymus serpyllum L.) on the copper-induced oxidation of human low-density lipoproteins. Int. J. Food Sci. Nutr. 2007; 58:87-93. [PubMed: 17469764]

3. Ogier N, Amiot M-J, Georgé S, Maillot M, Mallmann C, et al. LDL-cholesterol-lowering effect of a dietary supplement with plant extracts in subjects with moderate hypercholesterolemia. Eur. J. Nutr. 2013; 52:547-557. [PubMed: 22527287]

4. Mursu J, Voutilainen S, Nurmi T, Rissanen T-H, Virtanen J-K, et al. Dark chocolate consumption increases HDL cholesterol concentration and chocolate fatty acids may inhibit lipid peroxidation in healthy humans. Free Radic. Biol. Med. 2004; 37:1351-1359. [PubMed: 15454274] 
5. Sarriá B, Martínez-López S, Sierra-Cinos J-L, García-Diz L, Goya L, et al. Effects of bioactive constituents in functional cocoa products on cardiovascular health in humans. Food Chem. 2015; 174:214-218. [PubMed: 25529672]

6. Peñalvo J-L, López-Romero P. Urinary enterolignan concentrations are positively associated with serum HDL cholesterol and negatively associated with serum triglycerides in U.S. adults. J. Nutr. 2012; 142:751-756. [PubMed: 22378329]

7. Covas M-I, Nyyssönen K, Poulsen H-E, Kaikonnen J, Zunft H-J, et al. The effect of polyphenols in olive oil on heart disease risk factors. Ann. Intern. Med. 2006; 145:333-341. [PubMed: 16954359]

8. Gómez M, Vila J, Elosua R, Molina L, Bruguera J, et al. Relationship of lipid oxidation with subclinical atherosclerosis and 10-year coronary events in general population. Atherosclerosis. 2014; 232:134-140. [PubMed: 24401227]

9. Hernáez A, Fernández-Castillero S, Farràs M, Catalán U, Subirana I, et al. Olive oil polyphenols enhance high density lipoprotein function in humans. A randomized controlled trial. Arterioscler. Thromb. Vasc. Biol. 2014; 34:2115-2119. [PubMed: 25060792]

10. Farràs M, Valls R-M, Fernández-Castillejo $S$, Giralt M, Solà R, et al. Olive oil polyphenols enhance the expression of cholesterol efflux related genes in vivo in humans. A randomized controlled trial. J. Nutr. Biochem. 2013; 24:1334-1339. [PubMed: 23333095]

11. Otvos J-D. Measurement of lipoprotein subclass profiles by nuclear magnetic resonance spectroscopy. Clin. Lab. 2002; 48:171-180. [PubMed: 11934219]

12. Cole T-G, Contois J-H, Csako G, McConnell J-P, Remaley AT, et al. Association of apolipoprotein $\mathrm{B}$ and nuclear magnetic resonance spectroscopy-derived LDL particle number with outcomes in 25 clinical studies: assessment by the AACC Lipoprotein and Vascular Diseases Division Working Group on Best Practices. Clin. Chem. 2013; 59:752-770. [PubMed: 23386699]

13. Mackey R-H, Greenland P, Goff D-C Jr, Lloyd-Jones D, Sibley C-T, et al. High-density lipoprotein cholesterol and particle concentrations, carotid atherosclerosis, and coronary events: MESA (Multi-Ethnic Study of Atherosclerosis. J. Am. Coll. Cardiol. 2012; 60:508-516. [PubMed: 22796256]

14. Mora S, Glynn R-J, Ridker P-M. High-density lipoprotein cholesterol, size, particle number, and residual vascular risk after potent statin therapy. Circulation. 2013; 128:1189-1197. [PubMed: 24002795]

15. Parish S, Offer A, Clarke R, Hopewell J-C, Hill M-R, et al. Lipids and lipoproteins and risk of different vascular events in the MRC/BHF Heart Protection Study. Circulation. 2012; 125:24692478. [PubMed: 22539783]

16. Steffen B-T, Guan W, Remaley A-T, Paramsothy P, Heckbert S-R, et al. Use of lipoprotein particle measures for assessing coronary heart disease risk Post-American Heart Association/American Collegue of Cardiology guidelines. Arterioscler. Thromb. Vasc. Biol. 2015; 35:448-454. [PubMed: 25477346]

17. Qi Y, Fan J, Liu J, Wang W, Wang M, et al. Cholesterol-overloaded HDL particles are independently associated with progression of carotid atherosclerosis in a cardiovascular diseasefree population. J. Am. Coll. Cardiol. 2015; 65:355-363. [PubMed: 25634834]

18. Fielding C-J, Havel R-J, Todd K-M, Yeo K-E, Schloetter M-C, et al. Effects of dietary cholesterol and fat saturation on plasma lipoproteins in an ethnically diverse population of healthy young men. J. Clin. Invest. 1995; 95:611-618. [PubMed: 7860745]

19. Rubió L, Farrás M, de la Torre R, Maciá A, et al. Metabolite profiling of olive oil and thyme phenols after a sustained intake of two phenol-enriched olive oils by humans: Identification of compliance markers. Food Res. Int. 2014; 65:59-68.

20. Elosua R, Marrugat J, Molina L, Pons S, Pujol E. Validation of the Minnesota Leisure Time Physical Activity Questionnaire in Spanish men. The MARATHOM Investigators. Am. J. Epidemiol. 1994; 139:1197-1209. [PubMed: 8209878]

21. Elosua R, García M, Aguilar A, Molina L, Cobas M-I, et al. Validation of the Minnesota Leisure Time Physical Activity Questionnaire In Spanish Women. Med. Sci. Sports Exerc. 2000; 32:14311437. [PubMed: 10949009]

22. Jeyarajah E-J, Cromwell W-C, Otvos J-D. Lipoprotein particle analysis by nuclear magnetic resonance spectroscopy. Clin. Lab. Med. 2006; 26:847-870. [PubMed: 17110242] 
23. Shalaurova I, Connelly M-A, Garvey W-T, Otvos J-D. Lipoprotein Insulin Resistance Index: A Lipoprotein Particle-Derived Measure of Insulin Resistance. Metab. Syndr. Relat. Disord. 2014; 12:422-429. [PubMed: 24959989]

24. Blake G-J, Otvos J-D, Rifai N, Ridker P-M. Low-density lipoprotein particle concentration and size as determined by nuclear magnetic resonance spectroscopy as predictors of cardiovascular disease in women. Circulation. 2002; 106:1930-1937. [PubMed: 12370215]

25. El Harchaoui K, van der Steeg W-A, Stroes E-S, Kuivenhoven J-A, et al. Value of low-density lipoprotein particle number and size as predictors of coronary artery disease in apparently healthy men and women: the EPIC-Norfolk Prospective Population Study. J. Am. Coll. Cardiol. 2007; 49:547-553. [PubMed: 17276177]

26. Violi F, Loffredo L, Pignatelli P, Angelico F, Barimoccia S, et al. Extra virgin olive oil use is associated with improved post-prandial blood glucose and LDL cholesterol in healthy subjects. Nutr. Diabetes. 2015; 5:e172. [PubMed: 26192450]

27. Larsson S-C. Coffee, tea, cocoa and risk of stroke. Stroke. 2014; 45:309-314. [PubMed: 24326448]

28. Bravo L, Mateos R, Sarriá B, Baeza G, Lecumberri E, et al. Hypocholesterolaemic and antioxidant effects of yerba mate (Ilex paraguariensis) in high-cholesterol fed rats. Fitoterapia. 2014; 92:219229. [PubMed: 24291756]

29. Hernáez A, Remaley A-T, Farràs M, Fernández-Castillejo S, Subirana I, et al. Olive oil polyphenols decrease LDL concentrations and LDL atherogenicity in men in a randomized controlled trial. J. Nutr. 2015; 145:1692-1697. [PubMed: 26136585]

30. Bhakdi S, Dorweiler B, Kirchmann R, Torzewski J, Weise E, et al. On the pathogenesis of atherosclerosis: enzymatic transformation of human low density lipoprotein to an atherogenic moiety. J. Exp. Med. 1995; 182:1959-1971. [PubMed: 7500042]

31. Lyons T-J. Glycation and oxidation: a role in the pathogenesis of atherosclerosis. Am. J. Cardiol. 1993; 71:26B-31B.

32. EFSA Panel on Dietetic Products. Nutrition and allergies (NDA). Scientific opinion on the substantiation of health claims related to polyphenols in olive oil and protection of LDL particles from oxidative damage. EFSA Journal. 2011; 9:2033. [accessed on September,2015] Available from: http://www.efsa.europa.eu/en/efsajournal/pub/2033.htm.

33. Akinkuolie A-O, Paynter N-P, Padmanabhan L, Mora S. High density lipoprotein subclass heterogeneity and incident coronary heart disease. Circ. Cardiovasc. Qual. Outcomes. 2014; 7:5563. [PubMed: 24248942]

34. Otvos J-D, Collins D, Freedman D-S, Shalaurova I, Schaefer E-J, et al. Low-density lipoprotein and high-density lipoprotein particle subclasses predict coronary events and are favourably changed by gemfibrozil therapy in the Veterans Affairs High-Density Lipoprotein Intervention Trial. Circulation. 2006; 113:1556-1563. [PubMed: 16534013]

35. Martin S-S, Khokhar A-A, May H-T, Kulkarni K-R, Blaha M-K, et al. HDL cholesterol subclasses, myocardial infarction, and mortality in secondary prevention: the lipoprotein investigators collaborative. Eur. Heart J. 2015; 36:22-30. [PubMed: 24980493]

36. Joshi P-H, Toth P-P, Lirette S-T, Griswold M-E, Massaro J-M, et al. Association of high-density lipoprotein subclasses and incident coronary heart disease: the Jackson Heart and Framingham Offspring Cohort Studies. Eur. J. Prev. Cardiol. 2016; 23:41-49. [PubMed: 25062744]

37. Zeljkovic A, Vekic J, Spasojevic-Kalimanovska V, Jelic-Ivanovic Z, Bogavac-Stanojevic N, et al. LDL and HDL subclasses in acute ischemic stroke: prediction of risk and short-term mortality. Atherosclerosis. 2010; 210:548-554. [PubMed: 20022325]

38. Borggreve S-E, De Vries R, Dullaart R-P-F. Alterations in high-density lipoprotein metabolism and reverse cholesterol transport in insulin resistance and type 2 diabetes mellitus: role of lipolytic enzymes, lecithin:cholesterol acyltransferase and lipid transfer proteins. Eur. J. Clin. Invest. 2003; 33:1051-1069. [PubMed: 14636288]

39. Sankaranarayanan S, Oram J-F, Asztalos B-F, Vaughan A-M, Lund-Katz S, et al. Effects of acceptor composition and mechanism of ABCG1-mediated cellular free cholesterol efflux. J. Lipid Res. 2009; 50:275-284. [PubMed: 18827283] 
40. Farràs M, Castañer O, Martin-Pelaez S, Hernáez A, Schröder H, et al. Complementary phenolenriched olive oil improves HDL characteristics in hypercholesterolemic subjects. A randomized, double-blind, crossover, controlled trial. The VOHF study. Mol. Nutr. Food Res. 2015; 59:17581770. [PubMed: 26011257]

41. Remaley A-T. HDL cholesterol/HDL particle ratio. A new measure for HDL function? J. Am. Coll. Cardiol. 2015; 65:364-366. [PubMed: 25634835]

42. Khera A-V, Cuchel M, de la Llera-Moya M, Rodrigues A, Burke M-F, et al. Cholesterol efflux capacity, high-density lipoprotein function, and atherosclerosis. N. Engl. J. Med. 2011; 364:127135. [PubMed: 21226578]

43. Huang Y, Didonato J-A, Levison B-S, Schmitt D, Li L, et al. An abundant dysfunctional apolipoprotein A1 in human atheroma. Nat med. 2014; 20(2):193-203. [PubMed: 24464187]

44. Lee S, Birukov K-G, Romanoski C-E, Springstead J-R, Lusis A-J, et al. Role of phospholipid oxidation products in atherosclerosis. Circ Res. 2012; 111(6):778-799. [PubMed: 22935534]

45. Garvey W-T, Kwon S, Zheng D, Shaughnessy S, Wallace P, et al. Effects of insulin resistance and type 2 diabetes on lipoprotein subclass particle size and concentration determined by nuclear magnetic resonance. Diabetes. 2003; 52:453-462. [PubMed: 12540621]

46. Paynter N-P, Sesso H-D, Conen D, Otvos J-D, Mora S. Lipoprotein subclass abnormalities and incident hypertension in initially healthy women. Clin. Chem. 2011; 57:1178-1187. [PubMed: 21700954]

47. Einhorn D, Reaven G-M, Cobin R-H, Ford E, Ganda O-P, et al. American College of Endocrinology position statement on the insulin resistance syndrome. Endocr. Pract. 2003; 9:237252. [PubMed: 12924350] 


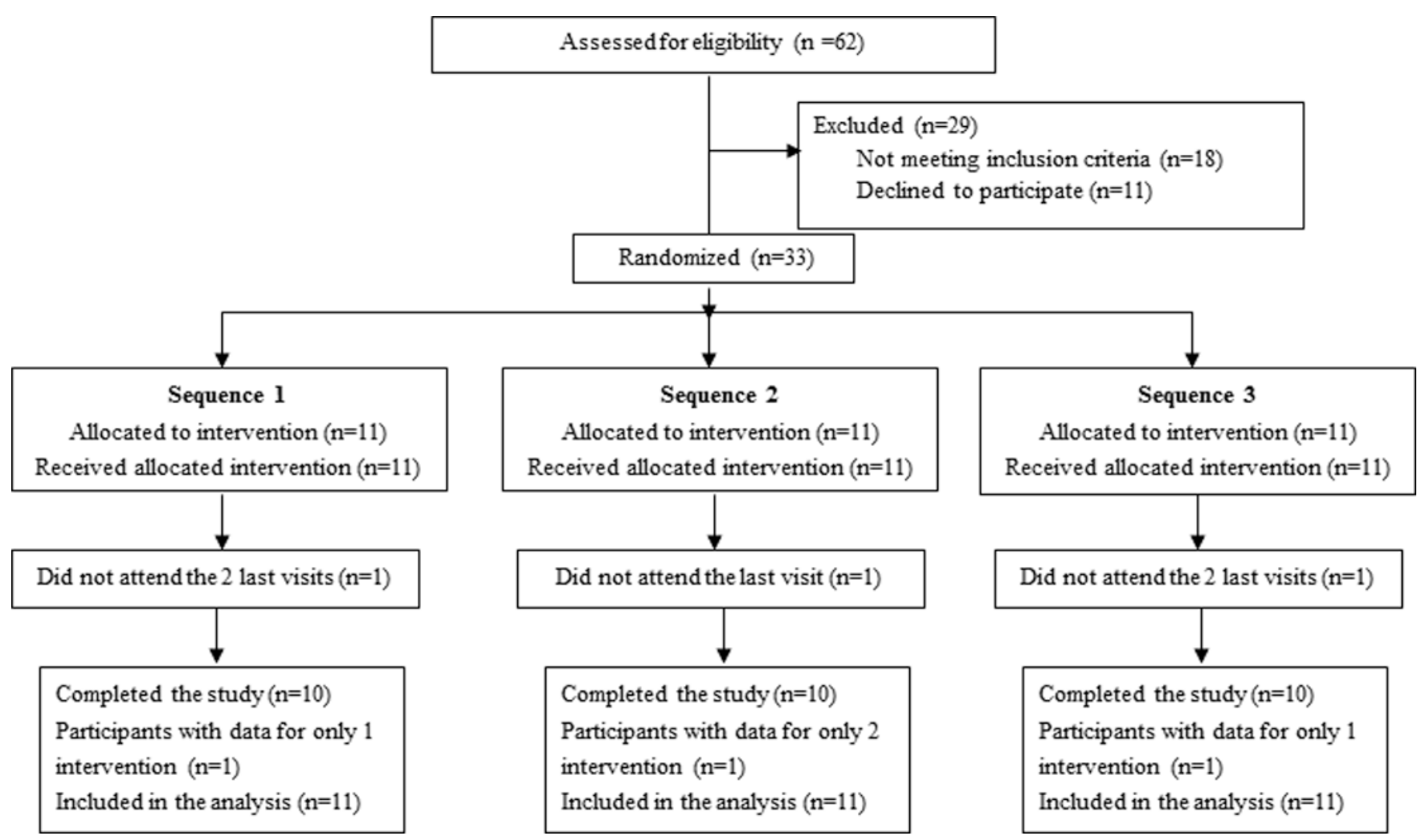

Figure 1.

Flow-chart of the study 
Hydroxytyrosol sulfate

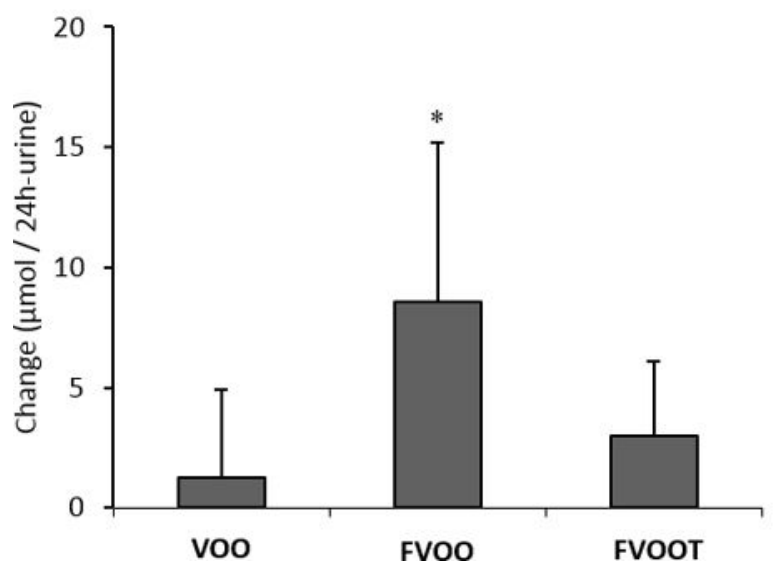

Thymol sulfate

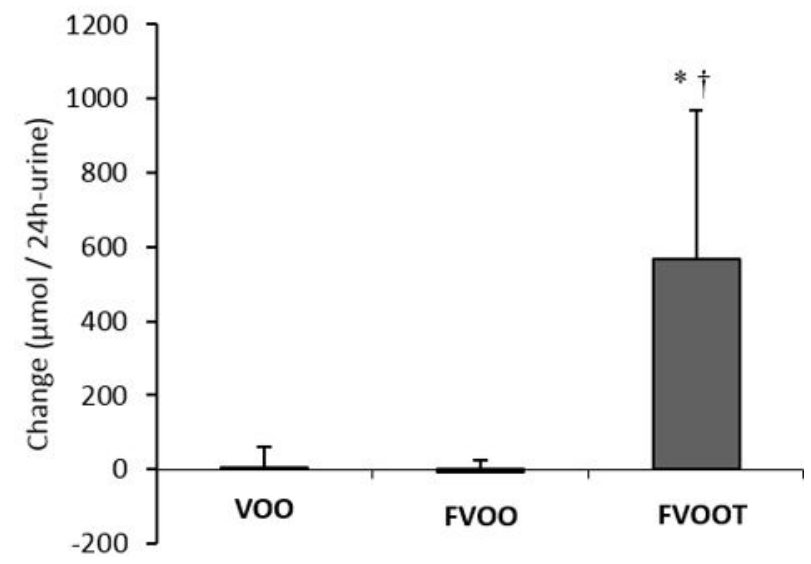

Figure 2.

Changes in urinary hydroxytyrosol sulfate and thymol sulfate after olive oil interventions.

VOO, virgin olive oil; FVOO, functional virgin olive oil enriched with its phenolic compounds (500 ppm); FVOOT, functional olive enriched with its phenolic compounds (250 ppm) and those from thyme (250 ppm). ${ }^{*} \mathrm{P}<0.05$ versus VOO; ${ }^{\dagger} \mathrm{P}<0.001$ versus FVOO. 


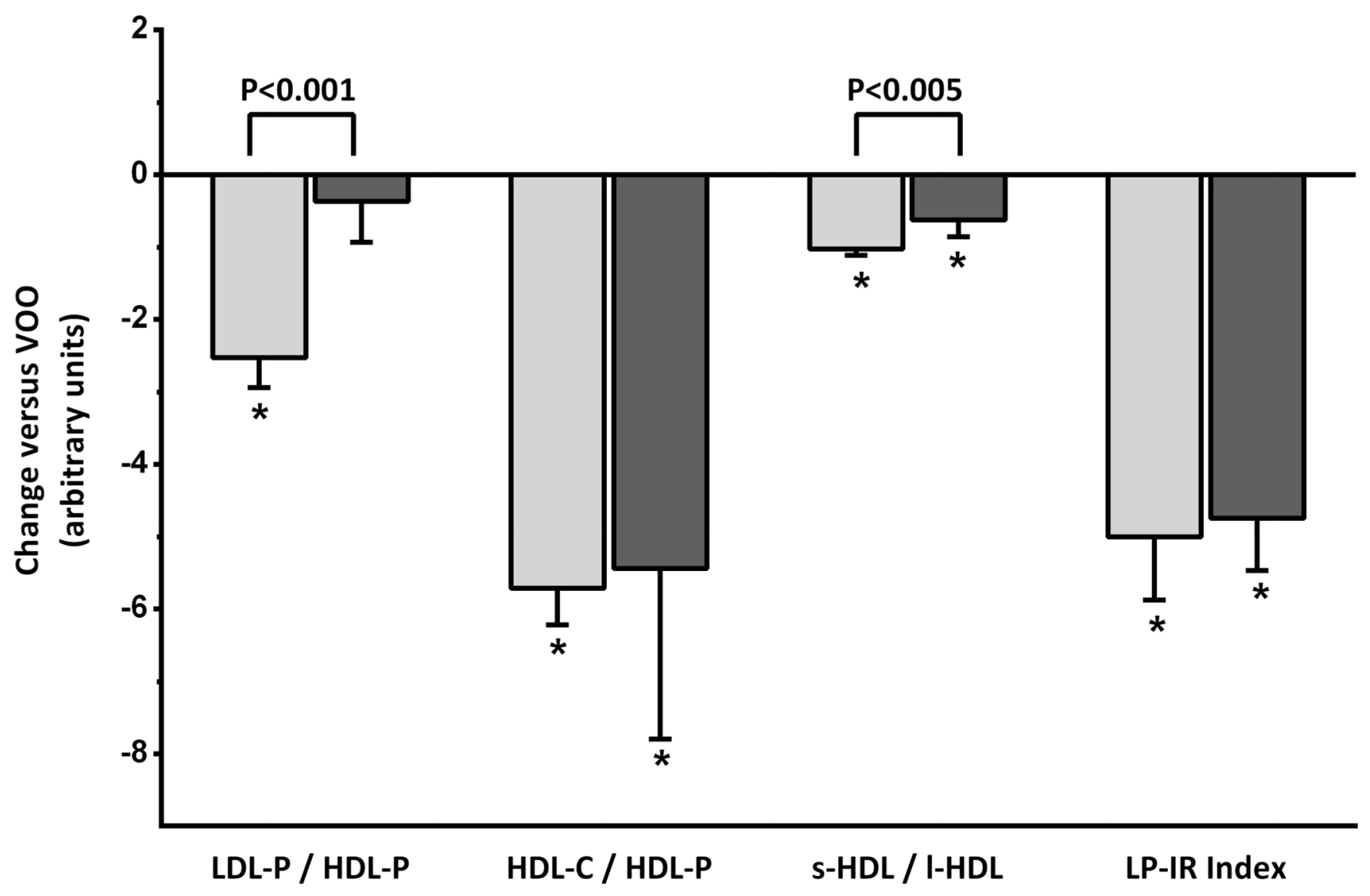

LL-P / HDL-P

HDL-C / HDL-P

SWL/I-HDL

FVOO vs VOO

FVOOT vs VOO

Figure 3.

Changes (mean \pm S.E.M) in atherogenic lipoprotein particle atherogenic ratios ratios and lipoprotein insulin resistance index (LP-IR) after consumption of functional olive oils versus natural virgin olive oil (VOO). FVOO, functional virgin olive oil enriched with its phenolic compounds (500 ppm); FVOOT, functional olive enriched with its phenolic compounds (250 $\mathrm{ppm}$ ) and those from thyme (250 ppm). LDL-P, low density lipoprotein particleconcentration; HDL-P, high density lipoprotein particle-concentration; HDL-C, HDL cholesterol; s-HDL, small HDL lipoprotein particle concentration; 1-HDL, large HDL lipoprotein particle concentration. ${ }^{*} \mathrm{P}<0.001$ versus VOO. Differences between functional olive oils are indicated by square brackets with the corresponding significance. 


\section{Table 1}

Baseline characteristics of the study participants.

\begin{tabular}{|c|c|c|c|}
\hline Variable & Sequence 1 & Sequence 2 & Sequence 3 \\
\hline Age (y) & $54.9 \pm 12.6$ & $55.3 \pm 11.9$ & $55.5 \pm 7.8$ \\
\hline Gender (male/female) & $5 / 6$ & $7 / 4$ & $7 / 4$ \\
\hline Body mass Index $\left(\mathrm{kg} / \mathrm{m}^{2}\right)$ & $25.6 \pm 3.7$ & $26.3 \pm 5.2$ & $27.8 \pm 4.7$ \\
\hline Physical activity (METs.min/w) & $\begin{array}{c}3499 \\
(1755-8092)\end{array}$ & $\begin{array}{c}1189 \\
(742-1687)\end{array}$ & $\begin{array}{c}3322 \\
(861-3664)\end{array}$ \\
\hline Systolic blood pressure (mmHg) & $125 \pm 18.7$ & $128 \pm 16.7$ & $130 \pm 17.9$ \\
\hline Diastolic blood pressure (mmHg) & $68.1 \pm 13.5$ & $72.3 \pm 9.3$ & $71.9 \pm 13.4$ \\
\hline Glucose (mg/dL) & $88.5 \pm 11.6$ & $93.0 \pm 13.3$ & $90.9 \pm 10.5$ \\
\hline Total cholesterol (mg/dL) & $228 \pm 42.7$ & $232 \pm 32.7$ & $219 \pm 31.2$ \\
\hline \multicolumn{4}{|l|}{ LDL measures } \\
\hline LDL cholesterol (mg/dL) & $150 \pm 32.3$ & $152 \pm 28.5$ & $142 \pm 25.7$ \\
\hline ApoB100 (mg/dL) & $115 \pm 0.21$ & $117 \pm 0.18$ & $109 \pm 0.16$ \\
\hline \multicolumn{4}{|l|}{ NMR LDL particle concentration (nmol/L) } \\
\hline Total & $1473 \pm 348$ & $1420 \pm 319$ & $1322 \pm 254$ \\
\hline IDL & $297 \pm 95.1$ & $295 \pm 165$ & $289 \pm 79.4$ \\
\hline Large & $593 \pm 139$ & $515 \pm 152$ & $433 \pm 227$ \\
\hline Small & $600 \pm 389$ & $560 \pm 381$ & $464 \pm 199$ \\
\hline Average NMR LDL particle size (nm) & $21.2 \pm 0.69$ & $21.1 \pm 0.71$ & $21.0 \pm 0.60$ \\
\hline \multicolumn{4}{|l|}{ VLDL measures } \\
\hline Triglycerides, mg/dL & $94(75-149)$ & $119(95-168)$ & $117(81-126)$ \\
\hline \multicolumn{4}{|l|}{ NMR VLDL particle concentration (nmol/L) } \\
\hline Total & $54.5 \pm 36.1$ & $65.6 \pm 26.6$ & $59.4 \pm 32.0$ \\
\hline Large & $3.8 \pm 4.6$ & $5.3 \pm 4.2$ & $2.8 \pm 2.1$ \\
\hline Medium & $16.2 \pm 11.5$ & $20.2 \pm 11.3$ & $16.8 \pm 10.7$ \\
\hline Small & $35.0 \pm 26.2$ & $39.6 \pm 16.5$ & $39.5 \pm 21.9$ \\
\hline Average NMRV VLDL particle size (nm) & $45.8 \pm 9.7$ & $48.2 \pm 5.5$ & $45.3 \pm 3.8$ \\
\hline $\begin{array}{l}\text { NMR ApoB100-containing particles } \\
\text { concentration }(\mathrm{nmol} / \mathrm{L})\end{array}$ & $1517 \pm 310$ & $1478 \pm 298$ & $1384 \pm 235$ \\
\hline \multicolumn{4}{|l|}{ HDL measures } \\
\hline HDL cholesterol (mg/dL) & $52.8 \pm 11.7$ & $53.0 \pm 12.8$ & $53.4 \pm 9.5$ \\
\hline ApoAI (mg/dL) & $142 \pm 0.23$ & $137 \pm 0.21$ & $147 \pm 0.16$ \\
\hline \multicolumn{4}{|l|}{ NMR HDL particle concentration $(\mu \mathrm{mol} / \mathrm{L})$} \\
\hline Total & $35.6 \pm 5.9$ & $34.6 \pm 5.3$ & $38.0 \pm 4.0$ \\
\hline Large & $7.0 \pm 3.8$ & $6.2 \pm 3.4$ & $6.6 \pm 2.6$ \\
\hline Medium & $13.0 \pm 6.2$ & $9.2 \pm 6.3$ & $9.3 \pm 4.7$ \\
\hline Small & $14.4 \pm 6.1$ & $18.0 \pm 5.2$ & $21.1 \pm 4.9^{*}$ \\
\hline Average NMR HDL particle size (nm) & $9.4 \pm 0.63$ & $9.3 \pm 0.54$ & $9.2 \pm 0.34$ \\
\hline
\end{tabular}

Values are expressed as means \pm SD or median (25th-75th percentile). Sequence 1: FVOO, FVOOT, VOO; Sequence 2: FVOOT, VOO, FVOO; Sequence 3: VOO, FVOO, FVOOT.

P $<0.05$ versus Sequence 1

Mol Nutr Food Res. Author manuscript; available in PMC 2017 July 01. 


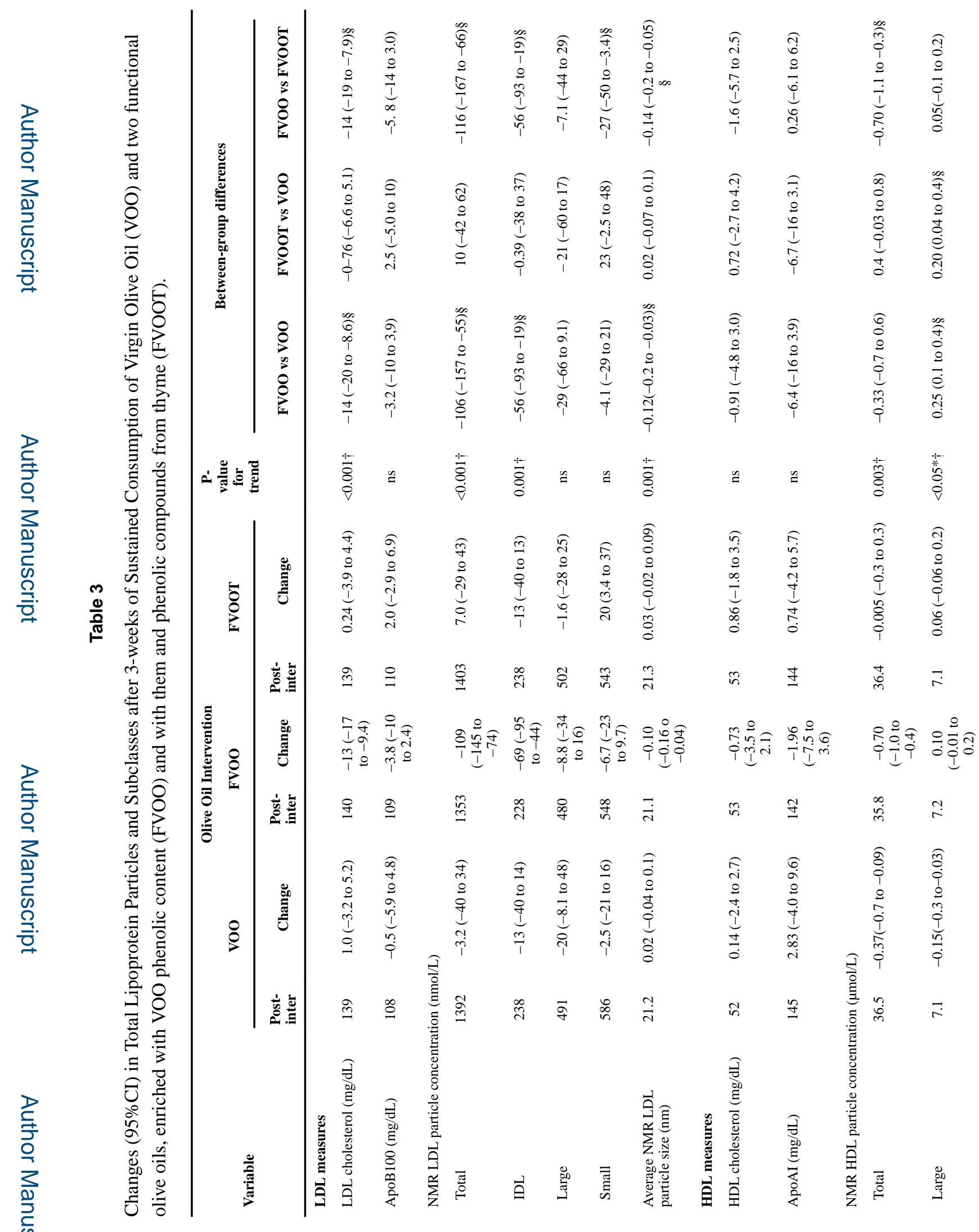

Mol Nutr Food Res. Author manuscript; available in PMC 2017 July 01. 


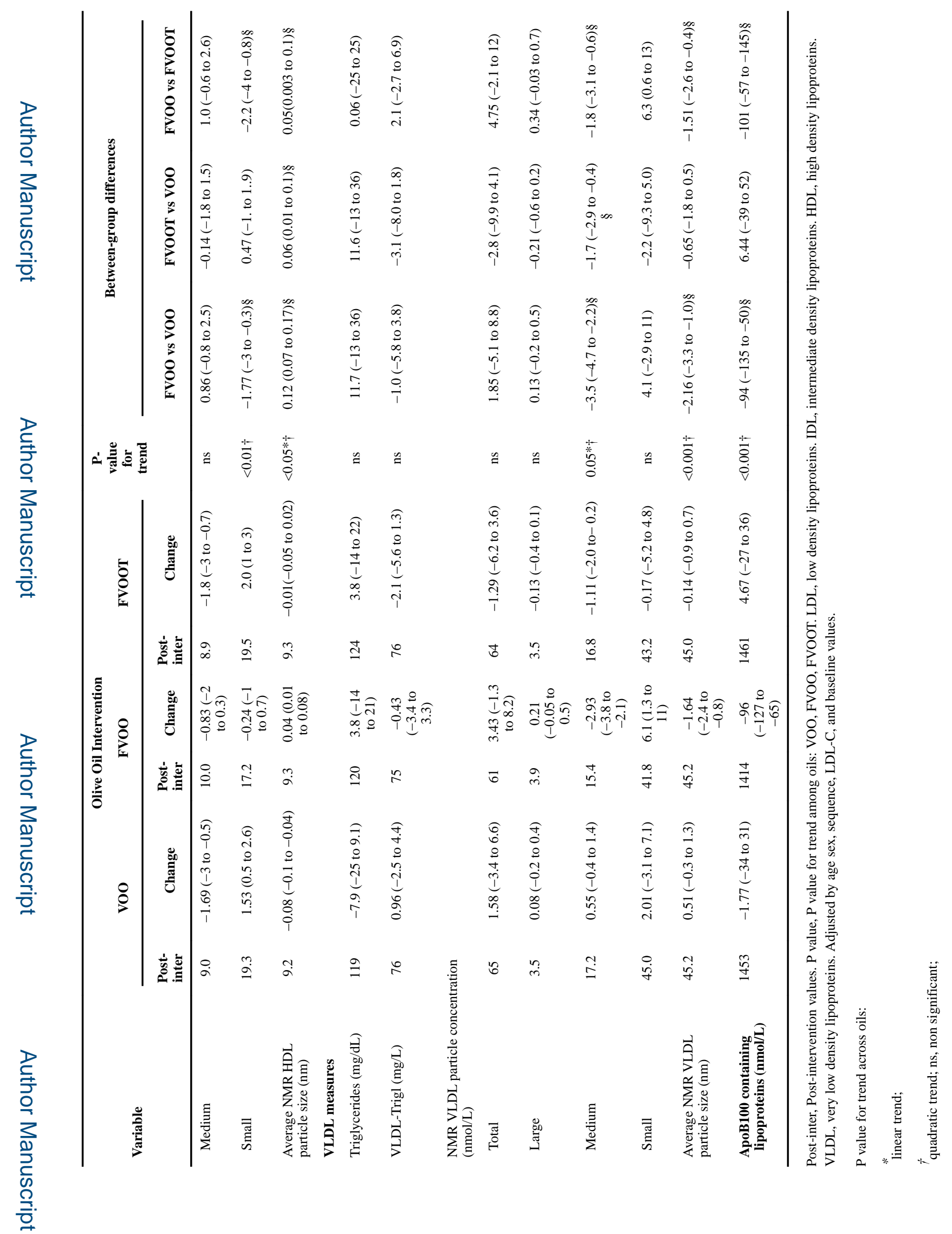

Mol Nutr Food Res. Author manuscript; available in PMC 2017 July 01. 
